\title{
Clinical trials using oncolytic viral therapy to treat adult glioblastoma: a progress report
}

\author{
Victor M. Lu, MD, PhD, Ashish H. Shah, MD, Frederic A. Vallejo, BS, Daniel G. Eichberg, MD, \\ Evan M. Luther, MD, Sumedh S. Shah, MD, Ricardo J. Komotar, MD, and Michael E. Ivan, MD, MBS \\ Department of Neurological Surgery, University of Miami Miller School of Medicine, Jackson Health System, Miami, Florida
}

OBJECTIVE Adult glioblastoma (GBM) has proven refractory to decades of innovation. Oncolytic viral therapy represents a novel therapy that uses viral vectors as both a delivery and therapeutic mechanism to target GBM cells. Despite the growing body of basic science data supporting the feasibility of viral therapy to treat GBM, the reporting of clinical trial results is heterogeneous. Correspondingly, the aim of this study was to present a contemporary summary of the progress all clinical trials have made to date.

METHODS The ClinicalTrials.gov database was reviewed in August 2020 for all possible interventional clinical trials involving viral vector-based therapy to treat adult GBM. These were then screened against selection criteria to identify pertinent clinical trials.

RESULTS A total of 29 oncolytic viral therapy trials treating adult GBM were identified. The median start and expected completion years were 2014 and 2020, respectively. At the time of this writing, 10 (35\%) trials were reported to have completed recruitment, whereas 7 (24\%) were actively recruiting. The median target enrollment number was 36 (range 13-108), with the majority of trials being phase I $(n=18,62 \%)$, and involving secondary GBM among other malignant glioma $(n=19,66 \%)$. A total of 10 unique viral vectors were used across all trials, with the most common being adenovirus $(n=16,55 \%)$. Only $2(7 \%)$ phase I trials to date have reported outcomes on the ClinicalTrials.gov portal. Results of 12 additional clinical trials were found in academic publications, with median progression-free and overall survival times of 3 and 15 months, respectively, after the first viral dose at recurrence. The coordination of the large majority of trials originated from the US $(n=21,72 \%)$, and the median number of testing sites per trial was 1 (range 1-15), via industry funding ( $n=18$ trials, 62\%).

CONCLUSIONS There are multiple early-stage oncolytic viral therapy clinical trials for adult GBM currently active. To date, limited results and outcomes are promising but scarce. The authors expect this to change in the near future because many trials are scheduled to have either nearly or actually reached their expected recruitment completion time. How exactly oncolytic viral therapy will fit into the current treatment paradigms for primary and secondary GBM remains to be seen, and will not be known until safety and toxicity profiles are established by these clinical trials.

https://thejns.org/doi/abs/10.3171/2020.11.FOCUS20860

KEYWORDS glioblastoma; viral therapy; adenovirus; clinical trial; oncolytic

$\mathrm{T}$ HE prognosis of adult glioblastoma (GBM) has proven refractory to multiple decades of innovation since triple therapy involving maximal safe resection, chemotherapy, and radiation therapy was established in 2005. ${ }^{1}$ Correspondingly, the need to consider novel therapies continues to grow.

Oncolytic viral therapy is one such option. It remains an experimental platform that takes advantage of a virus' biological ability to infiltrate, replicate, and lyse human cells. ${ }^{2}$ By means of advanced recombinant technologies, naturally occurring viruses can be genetically engineered to target specific tumor cells based on the proteins these cells express, and they become oncolytic in nature. ${ }^{3}$ There are many possible mechanisms by which these vectors can result in the death of tumor cells once delivered, and include direct tumor lysis, modification of tumor microenvironment, priming of immune responses, inhibiting tumorigenic vascularization, and promoting immunogenic

ABBREVIATIONS GBM = glioblastoma; HSV = herpes simplex virus; MoMLV = Moloney murine leukemia virus; NCDV = Newcastle disease virus; NCT = National Clinical Trial; OS = overall survival; PFS = progression-free survival. 
cell death. ${ }^{5}$ Indeed, in vitro glioma cells were one of the earliest tumor cells to validate this concept of a virus that was able to be engineered into an oncolytic virus with tumor cell-killing abilities. ${ }^{5}$ Since those early days, our biological understanding of viral therapy has grown exponentially, to the point where multiple clinical trials for multiple cancer types are active. ${ }^{6}$

Attention has turned to oncolytic viral therapy as a possible novel therapy that may confer a prognostic benefit to adult patients with GBM, particularly at recurrence. However, progress to date with respect to in-human clinical trials remains subject to highly heterogeneous reporting and result dissemination. Correspondingly, the aim of this study was to present a contemporary summary of the progress that all clinical trials have made to date.

\section{Methods \\ Search Strategy}

The search strategy was designed to capture all possible clinical trials with registration in both the US National Library of Medicine and ClinicalTrials.gov registry (https://clinicaltrials.gov/) investigating a viral therapy to treat GBM. The ClinicalTrials.gov site is a database of privately and publicly funded clinical trials on a wide range of diseases and conditions conducted around the world, providing information on more than 300,000 research studies conducted across all states of the US and in more than 200 countries worldwide. ${ }^{7,8}$ Databases were searched and screened in August 2020 using the following string of search terms for condition or disease: "glioblastoma" and "viral." Synonyms used were "childhood anaplastic astrocytoma/childhood high-grade cerebral astrocytoma/grade IV astrocytoma/Grade IV Astrocytic Tumor/WHO Grade IV Glioma" and "virus," respectively. For results of clinical trials, a combination of ClinicalTrials.gov, PubMed, and Google Scholar electronic academic search engines were used to look for viral vectors and National Clinical Trial (NCT) registration matching those described in the included studies.

\section{Selection Criteria}

For inclusion into our study, trials were required to investigate a brain tumor cohort with the following features: 1) intracranial GBM and/or malignant high-grade glioma as the only or major ( $>80 \%$ ) histological diagnosis, and 2) whose trial treatment involved a viral vector-based intervention designed to target and kill GBM cells as per the oncolytic viral therapy concept. This did not include interventions such as gene therapy that involved virus vectors for the purposes of white blood cell transformation. In the case of trials reporting outcomes that included gliomas of the spine, low-grade gliomas, or tumors of other organ systems outside the brain, these were not included due to lack of specificity and generalizability. Trials targeting the cytomegalovirus via dendritic cell therapy were not included because they did not use the virus as a therapeutic vector. Additionally, pediatric trials were also excluded to avoid biological confounding. There were no limitations set on age, nature of presentation (e.g., primary vs recurrence), or geographic location.
Assessment of clinical trials to satisfy these criteria was performed independently by two investigators (V.M.L. and F.A.V.), and any discrepancies were resolved by discussion.

\section{Data Outcomes}

The following outcomes were then extracted from the database: NCT number, title, institution of correspondence, country of origin of the corresponding institution, number of institutions involved, involvement of outside countries, recruitment status, availability of results, type of primary intervention, primary and secondary outcome measurements, gender enrollment, age of enrollment, number of patients enrolled, study phases, study type, funding source, start year, completion year, year of the first release of results, and last updated year. ${ }^{9}$ All missing data were reported as "not reported." All data analyses, including the generation of figures and tables, were performed using STATA version 14.1 (StataCorp).

\section{Results}

\section{Search Strategy}

The initial search of the portal yielded 52 trials for screening. Eleven were excluded because their intervention did not involve a primary viral vector, 7 were excluded as they were pediatric trials, 3 describing dendritic cell therapy targeting cytomegalovirus were excluded, and 2 were excluded because they involved tumors in multiple organ systems. Ultimately, 29 trials were identified as appropriate for inclusion in this study, with parameters of the trials summarized in Table 1, and all individual details in Table 2.

\section{Status}

All trials reported start years between 2001 and 2020 (Fig. 1A), with a median start year of 2014. The period of 2017-2020 alone included 12 (41\%) trials commencing recruitment. In terms of expected completion year, the median was 2020 (Fig. 1B). As of August 2020, 10 (35\%) trials were reported to have completed recruitment; 7 (24\%) are recruiting; 7 (24\%) are declared as active, not recruiting; and $1(3 \%)$ as not yet recruiting. A total of $4(14 \%)$ trials had been terminated, suspended, or withdrawn since their commencement.

\section{Cohort}

The target number of patients to enroll across all trials ranged from 13 to 108, with the median number of patients being 36. There were 19 (66\%) trials involving primary and secondary GBM and other malignant glioma, 5 (17\%) trials involving primary and secondary GBM only, and 5 (17\%) trials involving secondary GBM only. There were no trials involving primary GBM only.

\section{Trial Design}

The most common trials were $18(62 \%)$ phase I trials, followed by 5 (17\%) phase I and II trials, then 5 (17\%) phase II trials, and 1 (3\%) phase II and III trial (Fig. 2). Four trials reported a randomization process in their al- 
location design, in 1 phase I trial, 2 phase II trials, and 1 phase II and III trial. Allocation in all other trials was nonrandomized. The most common primary outcome of interest was safety and toxicity in $24(83 \%)$ trials, with progression-free survival (PFS), radiographic response, and immunological response the other outcomes reported.

\section{Viral Therapy}

Across all trials, 10 unique viral vectors were used (Fig. 3). The most common was adenovirus, used in 16 $(55 \%)$ trials. With respect to standard-of-care treatment, $21(72 \%)$ trials involved resection, 7 (24\%) trials involved chemotherapy, and 4 (13\%) trials involved radiation therapy.

\section{Trial Outcomes}

Only $2(7 \%)$ trials to date have reported outcomes on the ClinicalTrials.gov portal. NCT00390299 was a phase I trial investigating carcinoembryonic antigen-expressing measles virus (MV-CEA) in recurrent GBM. For their primary outcome, they reported that $6 / 9$ (67\%) patients experienced a grade $3+$ adverse event following viral therapy to a resection cavity versus $5 / 13(39 \%)$ patients following intratumoral administration status after stereotactic biopsy. NCT01491893 was a phase I safety study of an oncolytic polio/rhinovirus recombinant in recurrent GBM. For their primary outcomes, they reported their maximum tolerable dose of $10^{10}$ median tissue culture infectious dose $\left(\mathrm{TCID}_{50}\right)$ based on dose-limiting toxicity events in $60 \mathrm{pa}-$ tients. They also noted that the median survival in their trial cohort was 6 years.

\section{Survival Outcomes}

A systematic review of electronic academic search engines revealed results for 12 clinical trials outside the ClinicalTrials.gov portal, with 7 studies that described adenovirus vectors (NCT03679754,10 NCT02026271,11 NCT00031083, ${ }^{12} \quad$ NCT00589875, ${ }^{13} \quad$ NCT00870181, ${ }^{14}$ NCT02798406, ${ }^{15}$ NCT04406272 ${ }^{16}$ ), and then 1 study each describing outcomes of GBM using herpes simplex virus -1 (HSV-1; NCT00028158) ${ }_{17}^{17}$ Moloney murine leukemia virus (MoMLV; NCT02414165) ${ }_{1}^{18}$ Newcastle disease virus (NCDV; NCT01174537), ${ }^{19}$ parvovirus (NCT01301430), ${ }^{20}$ and reovirus (NCT00528684) ${ }^{21}$ viral vectors (Fig. 4). The median PFS from first viral dose was 3 months (range 1-8 months) across 8 trial results. The median overall survival (OS) from first viral dose was 15 months (range 11-32 months) across all these reported outcomes.

\section{Coordination and Funding}

The median number of sites per trial was 1 , with a range of 1-15 (Fig. 5A). A total of 7 countries are listed as the primary location for all included trials-the most common being the US with 21 (72\%) trials-with 7 (33\%) being coordinated from Massachusetts, 3 each from Texas and Alabama, 2 from Ohio, and 1 each from California, Illinois, Michigan, Minnesota, North Carolina, and Pennsylvania (Fig. 5B). Germany and Spain both had 2 (7\%) trials, which was the next most common number at a country level, followed by China, France, Israel, and the
TABLE 1. Summary of 29 clinical trial characteristics

\begin{tabular}{|c|c|}
\hline Parameter & Outcome* \\
\hline \multicolumn{2}{|l|}{ Time } \\
\hline Start yr & $2014(2001-2020)$ \\
\hline Expected completion yr & $2020(2003-2030)$ \\
\hline Results first posted & $2018,2020 \dagger$ \\
\hline Last updated & $2019(2005-2020)$ \\
\hline \multicolumn{2}{|l|}{ Status } \\
\hline \multicolumn{2}{|l|}{ Current status as of August 2020} \\
\hline Completed & $10(35 \%)$ \\
\hline Recruiting & $7(24 \%)$ \\
\hline Active, not recruiting & $7(24 \%)$ \\
\hline Withdrawn & $2(7 \%)$ \\
\hline Terminated & $1(3 \%)$ \\
\hline Suspended & $1(3 \%)$ \\
\hline Not yet recruiting & $1(3 \%)$ \\
\hline Study results available & $2(7 \%)$ \\
\hline \multicolumn{2}{|l|}{ Cohort } \\
\hline Target no. of patients enrolled & $36(13-108)$ \\
\hline \multicolumn{2}{|l|}{ GBM type } \\
\hline GBM only, primary & 0 \\
\hline GBM only, secondary & $5(17 \%)$ \\
\hline GBM only, primary \& secondary & $5(17 \%)$ \\
\hline GBM \& other malignant glioma & $19(66 \%)$ \\
\hline \multicolumn{2}{|l|}{ Design } \\
\hline \multicolumn{2}{|l|}{ Phase } \\
\hline I & $18(62 \%)$ \\
\hline |\& || & $5(17 \%)$ \\
\hline II & $5(17 \%)$ \\
\hline$\|$ \& III & $1(3 \%)$ \\
\hline \multicolumn{2}{|l|}{ Primary outcome of interest } \\
\hline Safety \& toxicity & $24(83 \%)$ \\
\hline PFS & $3(10 \%)$ \\
\hline Radiographic response & $2(7 \%)$ \\
\hline \multicolumn{2}{|l|}{ Location \& funding } \\
\hline \multicolumn{2}{|l|}{ Country of correspondence } \\
\hline US & $21(72 \%)$ \\
\hline Germany, Spain & 2 (7\% each) \\
\hline China, France, Israel, Netherlands & 1 (3\% each) \\
\hline No. of sites involved & $1(1-15)$ \\
\hline \multicolumn{2}{|l|}{ Funding } \\
\hline Industry & $18(62 \%)$ \\
\hline $\mathrm{NIH}$ & $7(24 \%)$ \\
\hline
\end{tabular}

${ }^{*}$ Continuous data reported as median (range) and categorical data reported as no. (\% total).

† Only 2 trials (NCT00390299 and NCT01491893) have reported results on the ClinicalTrials.gov portal.

Netherlands with 1 (3\%) each. The majority of funding was through industry in the case of $18(62 \%)$ trials, with only $7(24 \%)$ trials reporting national funding via the National Institutes of Health (NIH). 
TABLE 2. Individual characteristics of each clinical trial

\begin{tabular}{|c|c|c|c|c|c|c|c|c|c|c|c|c|}
\hline $\begin{array}{l}\text { Trial } \\
\text { No. }\end{array}$ & NCT No. & $\begin{array}{c}\text { Coordinating } \\
\text { Country }\end{array}$ & $\begin{array}{l}\text { Trial } \\
\text { Sites } \\
\text { (no.) }\end{array}$ & Phase(s) & $\begin{array}{c}\text { Enrollment } \\
\text { Target } \\
\text { (no.) }\end{array}$ & $\begin{array}{l}\text { Viral } \\
\text { Vector }\end{array}$ & $\begin{array}{l}\text { GBM } \\
\text { Only }\end{array}$ & GBM Type & $\begin{array}{c}\text { Start } \\
\mathrm{Yr}\end{array}$ & $\begin{array}{c}\text { Expected } \\
\text { Completion } \\
\text { Yr }\end{array}$ & Status & $\begin{array}{c}\text { Primary } \\
\text { Outcome of } \\
\text { Interest }\end{array}$ \\
\hline 1 & NCT03294486 & France & 1 & $|\&| \mid$ & 78 & Poxvirus & Yes & Secondary & 2017 & 2021 & Recruiting & Toxicity \\
\hline 2 & NCT01174537 & Israel & 1 & $|\&| \mid$ & NR & NCDV & No & Secondary & 2011 & 2011 & Withdrawn & PFS \\
\hline 3 & NCT01956734 & Spain & 1 & I & 31 & Adenovirus & Yes & Secondary & 2013 & 2017 & Completed & Toxicity \\
\hline 4 & NCT00390299 & US & 1 & 1 & 23 & Measles & No & Secondary & 2006 & 2019 & Completed & Toxicity \\
\hline 5 & NCT04406272 & US & 2 & II & 45 & Adenovirus & Yes & Both & 2020 & 2023 & $\begin{array}{c}\text { Not yet } \\
\text { recruiting }\end{array}$ & Toxicity \\
\hline 6 & NCT03714334 & Spain & 1 & I & 24 & Adenovirus & Yes & Secondary & 2018 & 2022 & Recruiting & Toxicity \\
\hline 7 & NCT04105374 & US & 1 & $\|\&\|$ & NR & MoMLV & No & Primary & 2020 & 2030 & Withdrawn & PFS \\
\hline 8 & NCT00028158 & Germany & 1 & $|\&| \mid$ & 65 & HSV & No & Secondary & 2001 & 2003 & Completed & Toxicity \\
\hline 9 & NCT01301430 & Germany & 1 & $|\&| \mid$ & 18 & Parvovirus & Yes & Both & 2011 & 2015 & Completed & Toxicity \\
\hline 10 & NCT04327011 & US & 11 & 1 & 65 & MoMLV & No & Both & 2011 & 2019 & Terminated & Toxicity \\
\hline 11 & NCT01811992 & US & 1 & 1 & 19 & Adenovirus & No & Primary & 2014 & 2021 & $\begin{array}{l}\text { Active, not } \\
\text { recruiting }\end{array}$ & Toxicity \\
\hline 12 & NCT02197169 & US & 4 & I & 37 & Adenovirus & No & Secondary & 2014 & 2018 & Completed & Radiographic \\
\hline 13 & NCT01582516 & Netherlands & 1 & $|\&| \mid$ & 20 & Adenovirus & Yes & Secondary & 2010 & 2014 & Completed & Toxicity \\
\hline 14 & NCT03657576 & US & 1 & I & 24 & HSV & No & Secondary & 2019 & 2024 & Recruiting & Toxicity \\
\hline 15 & NCT00870181 & China & 1 & II & 47 & Adenovirus & No & Both & 2008 & 2012 & Completed & PFS \\
\hline 16 & NCT02798406 & US & 15 & II & 49 & Adenovirus & No & Secondary & 2016 & 2021 & $\begin{array}{l}\text { Active, not } \\
\text { recruiting }\end{array}$ & Radiographic \\
\hline 17 & NCT02062827 & US & 1 & I & 36 & HSV & No & Both & 2014 & 2023 & Recruiting & Toxicity \\
\hline 18 & NCT03679754 & US & 4 & I & 36 & Adenovirus & Yes & Both & 2018 & 2021 & $\begin{array}{l}\text { Active, not } \\
\text { recruiting }\end{array}$ & Toxicity \\
\hline 20 & NCT03152318 & US & 2 & I & 108 & HSV & No & Both & 2017 & 2022 & Recruiting & Toxicity \\
\hline 21 & NCT02026271 & US & 6 & I & 48 & Adenovirus & No & Both & 2015 & 2019 & $\begin{array}{l}\text { Active, not } \\
\text { recruiting }\end{array}$ & Toxicity \\
\hline 24 & NCT04214392 & US & 1 & I & 36 & Lentivirus & No & Both & 2020 & 2023 & Recruiting & Toxicity \\
\hline 25 & NCT03636477 & US & 4 & I & 21 & Adenovirus & Yes & Both & 2018 & 2021 & $\begin{array}{l}\text { Active, not } \\
\text { recruiting }\end{array}$ & Toxicity \\
\hline 26 & NCT04006119 & US & 7 & II & 36 & Adenovirus & Yes & Both & 2019 & 2022 & $\begin{array}{l}\text { Active, not } \\
\text { recruiting }\end{array}$ & Toxicity \\
\hline 27 & NCT03896568 & US & 1 & I & 36 & Adenovirus & No & Both & 2019 & 2020 & Recruiting & Toxicity \\
\hline 28 & NCT01491893 & US & 1 & I & 61 & Rhinovirus & Yes & Secondary & 2012 & 2021 & $\begin{array}{l}\text { Active, not } \\
\text { recruiting }\end{array}$ & Toxicity \\
\hline 29 & NCT00589875 & US & 4 & II & 52 & Adenovirus & No & Both & 2007 & 2016 & Completed & Toxicity \\
\hline 30 & NCT00031083 & US & 5 & I & 35 & Adenovirus & No & Both & 2002 & 2004 & Suspended & Toxicity \\
\hline 31 & NCT03072134 & US & 2 & 1 & 13 & Adenovirus & No & Primary & 2017 & 2020 & Completed & Toxicity \\
\hline 32 & NCT00528684 & US & 3 & I & 18 & Reovirus & No & Both & 2006 & 2010 & Completed & Toxicity \\
\hline
\end{tabular}

$\mathrm{NR}=$ not reported.

\section{Discussion}

Adult GBM is a devastating diagnosis with no cure despite decades of research and clinical trials. The emergence of oncolytic viral therapy has given new hope for discovering impactful interventions that can improve quality of life and survival in these patients. Our interrogation of the ClinicalTrials.gov portal reveals that there are many trials ongoing, and that most remain in the early safety and toxicity phases of investigation. Furthermore, the data would suggest that many trials are scheduled to either have recently completed recruiting or will be completed within the coming years, stoking anticipation for meaningful results to emerge soon after. Then, the way in which oncolytic viral therapy could fit into the current treatment paradigm of triple therapy at initial diagnosis will need to be elucidated.

Clinical trials involving oncolytic viral therapy to target cancers have grown in number in recent years, with varying degrees of completion rates. ${ }^{22}$ Although the earliest reported trial for GBM in adults was listed to have 

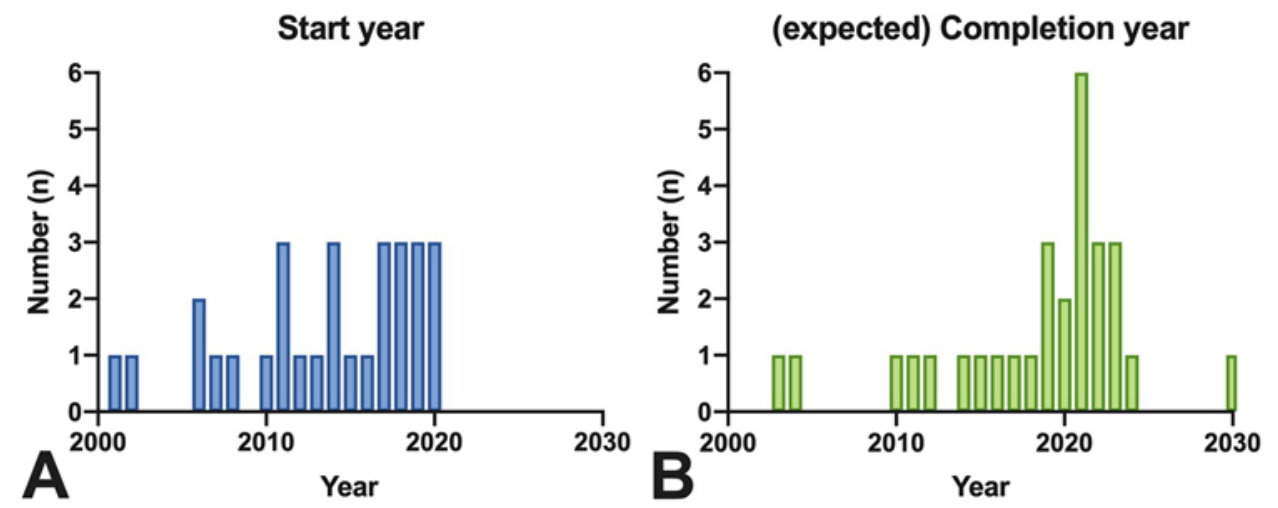

FIG. 1. Bar graphs showing the number of trials per start year $(\mathbf{A})$ and the completion year or expected completed year of recruitment (B).

commenced recruitment in 2001, the majority of the trials we report started in 2013 or later. However, estimated dates for recruitment completion remain largely contemporary, with less than half of all trials having completion status. Furthermore, very few trials have posted results of their study. A reason for these lags may be intrinsic to trial design, development, and ethics approval needed to commence and continue viral therapy investigation in patients with GBM. Additionally, we note that 4 trials have been terminated, suspended, or withdrawn since their commencement. Although it is pure speculation as to the reasons why this occurred, it does speak to the technical difficulty and perseverance required to take this novel therapy from bench to bedside. Multiple practical features such as success, costs, and expertise are likely to be factors requiring constant attention and revision in order to take these trials to completion. ${ }^{23}$

Although it is a novel treatment, emerging evidence in our review suggests that oncolytic viral therapy may confer a greater OS benefit compared to PFS benefit from

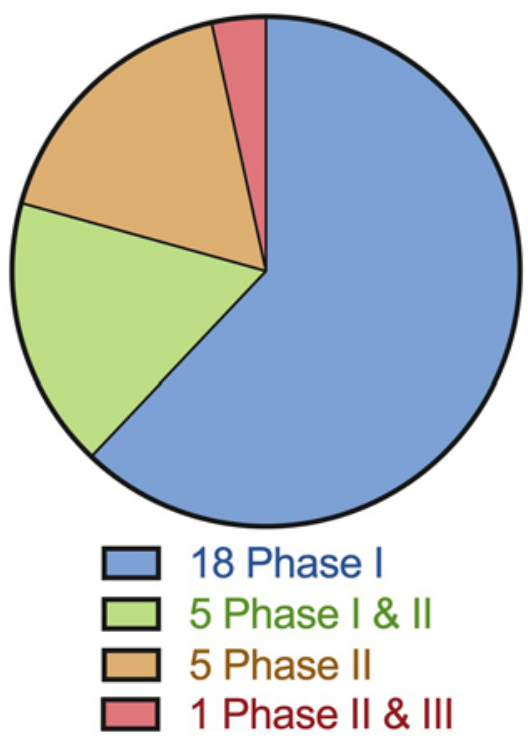

FIG. 2. Pie chart showing the distribution of clinical trials by phase. the start of the first dose. These findings are even more encouraging given that all studies incorporated patients with GBM who had recurrent disease, meaning overall time from initial diagnosis to recurrence must be added to these reported values for a true OS. It will be of great interest moving forward to see if these survival values extend as we accumulate more results and greater technical expertise.

Our results show that clinical trials of oncolytic viral therapy to treat adult patients with GBM are often singlesite studies involving fewer than 50 patients. Although part of this could be attributed to the relatively rare incidence of GBM, there are other trials investigating other novel therapies that have completed higher enrollment goals. For example, tumor-treating field technology has emerged as another novel modality to treat GBM in adults, and already there have been randomized trials ${ }^{24,25}$ involving hundreds of patients, as well as many other phase II and III trials with enrollment targets greater than 50 that are currently ongoing. ${ }^{26}$ In comparison, the majority of active clinical trials are phase I studies. Our findings then temper the expectation that novel therapies to treat GBM are directly comparable, in the sense that the biological variance of some treatments such as viral therapy demand

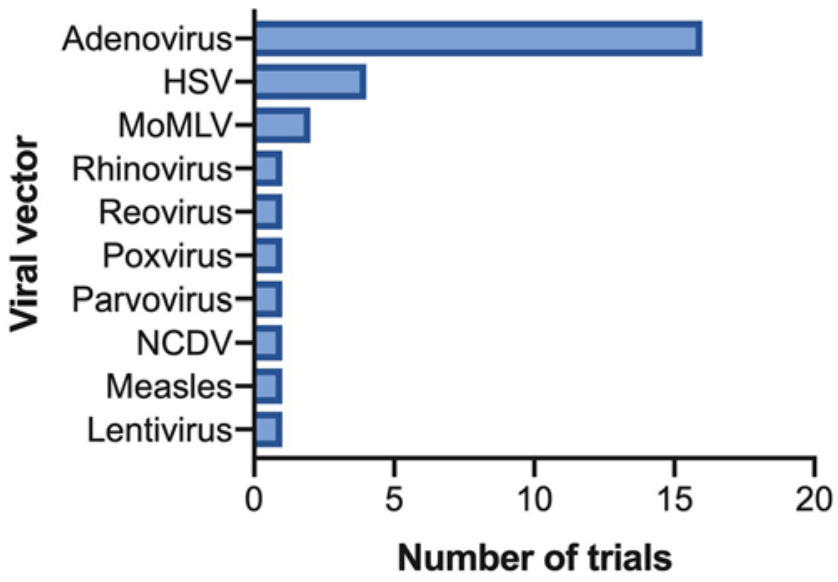

FIG. 3. Summary of the viral vectors used in each trial. 


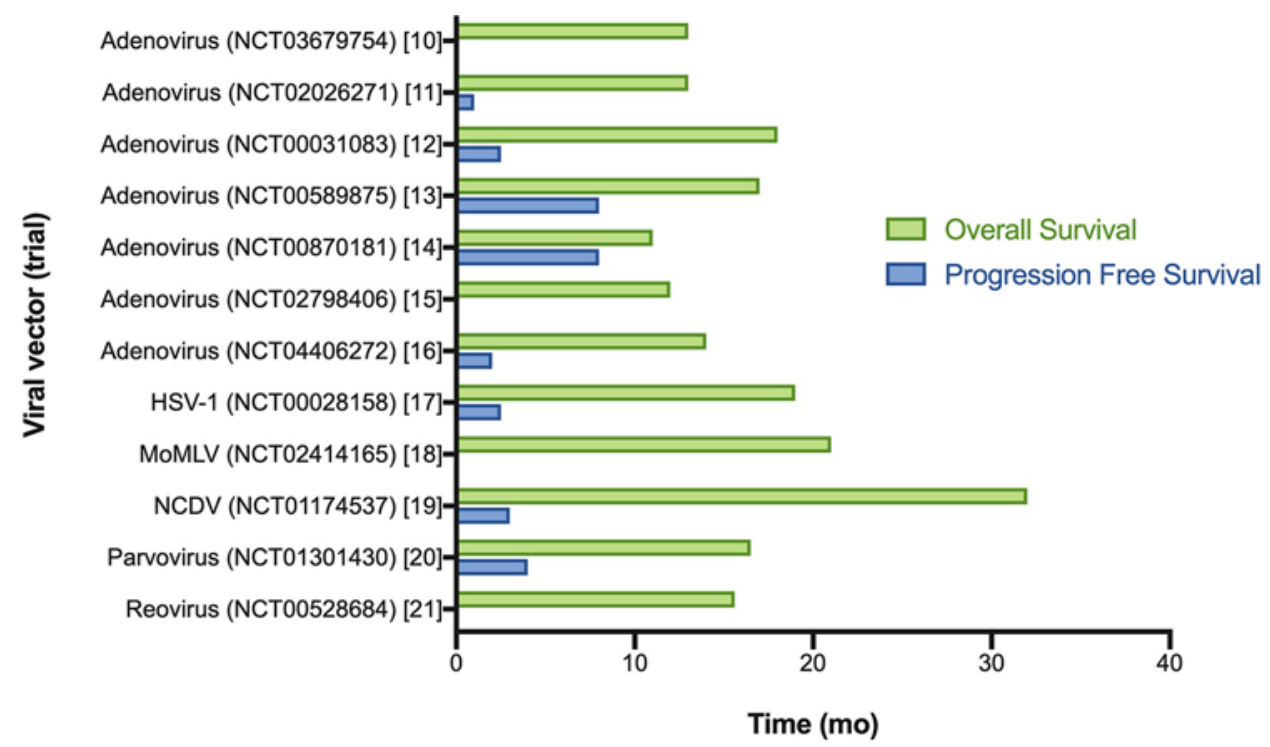

FIG. 4. Reported median OS and PFS of pertinent clinical trials. The NCT identifier is included in parentheses following the viral vector. The academic reference is listed in square brackets.

greater time and titration than other novel treatments like the tumor-treating field modality.

There are a large number of different viral vectors currently being investigated in GBM clinical trials as an oncolytic virus medium, with the most common being adenovirus. This is not particularly surprising given that the adenovirus is the most common viral vector used across all cancers, and it was the first vector to be used in a trial in human patients. ${ }^{3,27}$ The next most common viral vector was that of HSV, the same vector used in the original in vitro experiments with glioma. ${ }^{5}$ We note that to date no clinical trial is directly comparing multiple different viral vectors against each other, which may be due to the prematurity of assessing clinical impact at this current stage of development. Nonetheless, this vast array of potential vector choices currently being investigated across all clinical trials demonstrates the large biological variance that viral therapy development faces in its optimization. ${ }^{28}$

No clinical trial that we report was found to involve only primary GBM in its study cohort_rather, all trials in- cluded patients with recurrent GBM, for which the optimal treatment sequence other than repeat surgery remains unclear. ${ }^{29}$ The reason this could be an issue moving forward is that it will be difficult to discern efficacy potential from a recurrent GBM standpoint alone due to the large clinical heterogeneity in care at this point of diagnosis. Although the majority of trials involve repeat resection of recurrent GBM when evaluating viral therapy, only a minority included chemotherapy and radiation therapy. Direct comparability between active trials would therefore appear to be unwise. Even if modalities could be matched, however, viral therapies will face the challenge of how delivery affects their efficacy, with it being possible to deliver viral vectors to the tumor site via convection-enhanced delivery (NCT04214392), local injection at time of biopsy or resection (NCT01956734), or even systemically before or after surgery (NCT00870181).

There are limitations to this study. First, although the ClinicalTrials.gov portal is a comprehensive database, it is based in the US and is not an exhaustive list of every
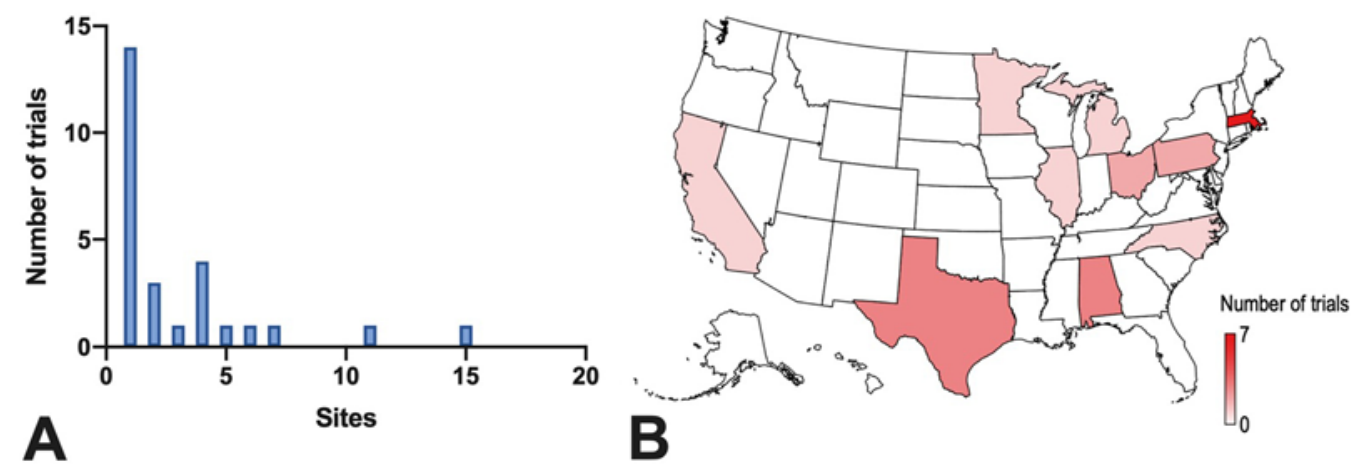

FIG. 5. Summary of the coordination of trials from the number of sites per trial (A), and distribution of the coordination sites by US state $(B)(n=21)$. 
single clinical trial being conducted in the world. ${ }^{30}$ There will probably be some trials that have not been registered on this portal, and indeed, as our systematic search of electronic academic search engines shows, not all results are always reported on the portal itself, but rather in academic publications. However, we suspect that the proportions of trials complete and those with results reported are congruent with contemporary efforts worldwide. Second, granular details as to the motivation, direction, and rationale of each clinical trial cannot be abstracted based on the data provided at hand. Understanding the selection of oncolytic viral vector and the decision to pursue specific evaluations would greatly assist in the broader development and uptake of this novel therapy. Finally, the lack of posted results limits our ability to make inferences on how exactly viral therapy may clinically impact management of GBM in adults in the future. The distinct lack of trials involving primary GBM perhaps underestimates the clinical potential and significance of this novel therapy. This is because there are a number of other emerging novel therapies being tested in the sphere of secondary GBM, limiting the time and resources that we as a wider community can give to this specific therapy and to discovering its true efficacy.

\section{Conclusions}

Oncolytic viral therapy to treat adult patients with GBM is a novel concept. The basic science of using viral vectors to target GBM cells has been robustly developed over time, and we now find ourselves at the precipice of the advance to clinical translation into human patients. Our interrogation of a large clinical trials database reveals that a number of clinical trials are active and are investigating the safety and toxicity profile of a number of different viral vectors. Results are largely pending, and are expected to be complete and hopefully posted within the coming years. Understanding this will then arm clinicians and scientists around the world with sufficient data to design effective higher-phase trials to test efficacy. Ultimately, future clinical trials based on the outcomes of current trials are needed to validate the place oncolytic viral therapy warrants among current treatment regimens to improve the prognosis of this dire disease.

\section{References}

1. Stupp R, Mason WP, van den Bent MJ, et al. Radiotherapy plus concomitant and adjuvant temozolomide for glioblastoma. N Engl J Med. 2005;352(10):987-996.

2. Aghi M, Martuza RL. Oncolytic viral therapies-the clinical experience. Oncogene. 2005;24(52):7802-7816.

3. Zheng M, Huang J, Tong A, Yang H. Oncolytic viruses for cancer therapy: barriers and recent advances. Mol Ther Oncolytics. 2019;15:234-247.

4. Harrington K, Freeman DJ, Kelly B, et al. Optimizing oncolytic virotherapy in cancer treatment. Nat Rev Drug Discov. 2019;18(9):689-706

5. Martuza RL, Malick A, Markert JM, et al. Experimental therapy of human glioma by means of a genetically engineered virus mutant. Science. 1991;252(5007):854-856.

6. Fukuhara H, Ino Y, Todo T. Oncolytic virus therapy: a new era of cancer treatment at dawn. Cancer Sci. 2016;107(10): 1373-1379.
7. Pfiffner PB, Oh J, Miller TA, Mandl KD. ClinicalTrials.gov as a data source for semi-automated point-of-care trial eligibility screening. PLoS One. 2014;9(10):e111055.

8. Huser V, Cimino JJ. Linking ClinicalTrials.gov and PubMed to track results of interventional human clinical trials. PLoS One. 2013;8(7):e68409.

9. Lim KJ, Yoon DY, Yun EJ, et al. Characteristics and trends of radiology research: a survey of original articles published in AJR and Radiology between 2001 and 2010. Radiology. 2012;264(3):796-802.

10. Chiocca EA, Lukas R, Yu J, et al. ATIM-15. A phase 1 study of Ad-RTS-hIL-12 + veledimex in adults with recurrent glioblastoma: dose determination with updated overall survival. Neuro Oncol. 2018;20(suppl 6):vi3-vi4.

11. Chiocca EA, Yu JS, Lukas RV, et al. Regulatable interleukin-12 gene therapy in patients with recurrent high-grade glioma: results of a phase 1 trial. Sci Transl Med. 2019; 11(505):eaaw5680.

12. Chiocca EA, Smith KM, McKinney B, et al. A phase I trial of Ad.hIFN- $\beta$ gene therapy for glioma. Mol Ther. 2008;16(3): $618-626$

13. Wheeler LA, Manzanera AG, Bell SD, et al. Phase II multicenter study of gene-mediated cytotoxic immunotherapy as adjuvant to surgical resection for newly diagnosed malignant glioma. Neuro Oncol. 2016;18(8):1137-1145.

14. Ji N, Weng D, Liu C, et al. Adenovirus-mediated delivery of herpes simplex virus thymidine kinase administration improves outcome of recurrent high-grade glioma. Oncotarget. 2016;7(4):4369-4378.

15. Aiken R, Chen C, Cloughesy T, et al. ATIM-33. Interim results of a phase II multi-center study of oncolytic adenovirus DNX-2401 with pembrolizumab for recurrent glioblastoma; captive study (Keynote-192). Neuro Oncol. 2019;21(suppl 6): vi8-vi9.

16. Brenner AJ, Peters KB, Vredenburgh J, et al. Safety and efficacy of VB-111, an anticancer gene therapy, in patients with recurrent glioblastoma: results of a phase I/II study. Neuro Oncol. 2020;22(5):694-704.

17. Markert JM, Razdan SN, Kuo H-C, et al. A phase 1 trial of oncolytic HSV-1, G207, given in combination with radiation for recurrent GBM demonstrates safety and radiographic responses. Mol Ther. 2014;22(5):1048-1055.

18. Cloughesy TF, Landolfi J, Vogelbaum MA, et al. Durable complete responses in some recurrent high-grade glioma patients treated with Toca 511 + Toca FC. Neuro Oncol. 2018; 20(10):1383-1392.

19. Freeman AI, Zakay-Rones Z, Gomori JM, et al. Phase I/II trial of intravenous NDV-HUJ oncolytic virus in recurrent glioblastoma multiforme. Mol Ther. 2006;13(1):221-228.

20. Geletneky K, Huesing J, Rommelaere J, et al. Phase I/IIa study of intratumoral/intracerebral or intravenous/intracerebral administration of Parvovirus H-1 (ParvOryx) in patients with progressive primary or recurrent glioblastoma multiforme: ParvOryx01 protocol. BMC Cancer. 2012;12:99.

21. Samson A, Scott KJ, Taggart D, et al. Intravenous delivery of oncolytic reovirus to brain tumor patients immunologically primes for subsequent checkpoint blockade. Sci Transl Med. 2018;10(422):eaam7577.

22. Zhang Y, Liu Z. Oncolytic virotherapy for malignant tumor: current clinical status. Curr Pharm Des. 2019;25(40):42514263.

23. Goradel NH, Baker AT, Arashkia A, et al. Oncolytic virotherapy: challenges and solutions. Curr Probl Cancer. Published online August 15, 2020. doi:10.1016/j.currproblcancer.2020.100639

24. Stupp R, Taillibert S, Kanner A, et al. Effect of tumor-treating fields plus maintenance temozolomide vs maintenance temozolomide alone on survival in patients with glioblastoma: a randomized clinical trial. JAMA. 2017;318(23):2306-2316. 
25. Stupp R, Wong ET, Kanner AA, et al. NovoTTF-100A versus physician's choice chemotherapy in recurrent glioblastoma: a randomised phase III trial of a novel treatment modality. Eur J Cancer. 2012;48(14):2192-2202.

26. Fabian D, Guillermo Prieto Eibl MDP, Alnahhas I, et al. Treatment of glioblastoma (GBM) with the addition of tumor-treating fields (TTF): a review. Cancers (Basel). 2019;11(2):E174.

27. Ganly I, Kirn D, Eckhardt G, et al. A phase I study of Onyx015, an E1B attenuated adenovirus, administered intratumorally to patients with recurrent head and neck cancer. Clin Cancer Res. 2000;6(3):798-806.

28. Eager RM, Nemunaitis J. Clinical development directions in oncolytic viral therapy. Cancer Gene Ther. 2011;18(5): 305-317.

29. Lu VM, Jue TR, McDonald KL, Rovin RA. The survival effect of repeat surgery at glioblastoma recurrence and its trend: a systematic review and meta-analysis. World Neurosurg. 2018;115:453-459.e3.

30. Stergiopoulos S, Getz KA, Blazynski C. Evaluating the completeness of ClinicalTrials.gov. Ther Innov Regul Sci. 2019; 53(3):307-317.

\section{Disclosures}

Dr. Ivan is a consultant for Medtronic.

\section{Author Contributions}

Conception and design: Lu, Vallejo, Luther. Acquisition of data: Lu, AH Shah, Vallejo, Luther. Analysis and interpretation of data: all authors. Drafting the article: all authors. Critically revising the article: all authors. Reviewed submitted version of manuscript: Lu, AH Shah, Vallejo, Eichberg, Luther, SS Shah, Komotar. Approved the final version of the manuscript on behalf of all authors: Lu. Statistical analysis: Lu. Study supervision: Ivan.

\section{Correspondence}

Victor M. Lu: University of Miami Miller School of Medicine, Miami, FL.victor.lu@jhsmiami.org. 